\title{
A few good reasons to consider a beam finite element formulation on the Lie group $S E(3)$
}

\author{
Valentin Sonneville and Olivier Brüls \\ Department of Aerospace and Mechanical Engineering (LTAS) \\ University of Liège \\ Chemin des Chevreuils 1, 4000 Liège, Belgium \\ Email: v.sonneville,o.bruls@ulg.ac.be
}

\begin{abstract}
Résumé
Based on an original interpolation method we develop a beam finite element formulation on the Lie group $S E(3)$ which relies on a mathematically rigorous framework and provides compact notations. We work out the beam kinematics in the SE(3) context, the beam deformation measure and obtain the expression of the internal forces using the virtual work principle. The proposed formulation exhibits important features from both the theoretical and numerical points of view. The approach leads to a natural coupling of position and rotation variables and thus differs from classical Timoshenko/Cosserat formulations. We highlight several important properties such as a constant deformation measure over the element, an invariant tangent stiffness matrix under of rigid motions or the absence of shear locking.
\end{abstract}

\section{INTRODUCTION}

The fundamental relationships in structural mechanics involve quantities that are based on position variables with respect to an inertial frame and its derivatives. For instance, the potential energy in a gravitational field depends on the position, the kinetic energy depends on the time derivative of the position, that is the velocity, and deformation energy depends on the spatial derivatives of the position, namely the deformation gradient. In order to describe particular bodies kinematic assumptions are made on the position of points of a body. This can be conveniently made by introducing rotation variables which account for some orientation. For example rotation variables can be used to describe the motion of a rigid body [1] or of a cross-section of a beam [2]. In this article we use this kind of method but notice that there are other methods which do not introduce any rotation field [3]. A sound comparison can be found in [4]. The development of the equations of mechanics under these assumptions leads in general to coupled differential equations that govern both the rotation and the position variables. The rotations belong to a non-linear space of variables 
such that their handling in general is not trivial. Several methods have been explored to deal with this such as the parametrization of rotation [1], the director vectors [5, 6] or the Lie group methods $[2,7,8,9]$. In this paper we consider Lie group methods. These methods are based on the differential geometry of the non-linear configuration space. The Lie group $\mathbb{R}^{3} \times S O(3)$ has been widely used for rigid body or beam formulations. Here, we consider the Lie group $S E(3)=\mathbb{R}^{3} \rtimes S O(3)$ which has been already applied to rigid body problems, in particular in robotic applications [10].

In this paper we develop a straight beam finite element formulation. In the finite element context a spatial discretization of the beam is introduced by an interpolation method of nodal values in order to obtain a discrete problem from a variational principle. The representation of the rotations by interpolation of the nodal rotation variables is a non-trivial issue due to the non-linearity of the configuration space. For example, Cardona and Géradin [11] and Simo [2] defined an interpolation method based on the increments of the rotations and Crisfield and Jelenic [12] proposed to interpolate the relative rotation matrix. In every case, the positions and the rotations are interpolated separately since they are considered to be fundamentally independent. In contrast, the $S E(3)$ Lie group framework of this paper allows to introduce a natural coupling of the position and the rotation variables thanks to an exponential interpolation method and exhibits important theoretical and numerical advantages. Let us mention that interpolation method in the $S E(3)$ framework have been addressed by [13] and [14] for motion interpolation of rigid bodies.

The paper is structured as follow. Firstly, some fundamentals about matrix Lie groups are given in order to introduce $S E(3)$ and the notations. Next, we consider the formulation of a static beam finite element. In particular, we work out the beam kinematics in the $S E(3)$ context, the beam deformation measure based on the proposed exponential interpolation formula and obtain the expression of the internal forces using the virtual work principle. Thirdly, we discuss some remarkable features of the approach in a few academic examples. Finally, some conclusions and perspectives are presented.

\section{FUNDAMENTALS ABOUT MATRIX LIE GROUPS}

We introduce here only the necessary concepts required for our developments. For a more general introduction to Lie groups see e.g. [10] or [15].

A group is a set of elements with a composition rule, that associates an element of the group to two elements of the group : if $q_{1}, q_{2} \in G$, then $q_{1} \circ q_{2}=q_{3} \in G$. In the present case we consider matrix groups and the composition rule is the classical matrix product written as $q_{1} q_{2}=q_{3}$. This composition rule has several properties as the existence of a neutral element $e(q e=e q=q)$, which is simply the identity matrix, and the existence of an inverse $\left(\forall q \in G, \exists ! q^{-1}: q q^{-1}=q^{-1} q=e\right)$. It follows that the elements of such a matrix group are square and invertible matrices. A matrix Lie group is a continuous matrix group for which the composition rule and the inverse are smooth. Therefore a matrix Lie group is 
geometrically speaking a differentiable manifold, and differential geometry can be used to perform operations on the group.

The composition operation can be seen as a particular map called the left translation map

$$
L_{y}(q): G \rightarrow G, q \rightarrow y q
$$

The tangent space at $q$ is a vector space and is denoted $T_{q} G$. The notation $\delta(\bullet)$ means an arbitrary infinitesimal variation of the argument, and thus $\delta q$ is an arbitrary element belonging to $T_{q} G$. Similarly, $\delta\left(L_{y}(q)\right)$ belongs to $T_{y q} G$, the tangent space at $y q$. The definition (1) yields a relationship between the tangent spaces $T_{q} G$ and $T_{y q} G$

$$
\delta\left(L_{y}(q)\right)=L_{*}(\delta q)=y \delta q
$$

where $L_{*}: T_{q} G \rightarrow T_{y q} G$ is an invertible linear map. The particular case $y=q^{-1}$ leads to an interesting fact : the tangent space of any $q \in G$ is related to the tangent space at the identity element $e$ through a linear map. The tangent space at the identity of a Lie group is called the Lie algebra $\mathfrak{g}$. It is isomorphic to $\mathbb{R}^{k}$ through the invertible linear map

$$
\widetilde{(\bullet)}: \mathbb{R}^{k} \rightarrow \mathfrak{g}, \mathbf{x} \rightarrow \widetilde{\mathbf{x}}
$$

Using these notations in Eqn. (2), we obtain

$$
\delta q=q \widetilde{\delta \mathbf{q}}
$$

Notice the difference in the two following notations : $\widetilde{\delta \mathbf{q}}$ and $\delta(\widetilde{\mathbf{q}})$. The former indicates an infinitesimal increment belonging to the Lie algebra, while the latter means the variation of an element $\widetilde{\mathbf{q}}$ of the Lie algebra. Similarly, $\delta \mathbf{q}$ and $\delta(\mathbf{q})$ stand for the associated expressions in $\mathbb{R}^{k}$ of respectively the former and the latter notation. The derivative of $q$ with respect to any parameter $s \in \mathbb{R}$ is written

$$
\frac{d q}{d s}=q \widetilde{\mathbf{u}}
$$

where $\tilde{\mathbf{u}}$ is an element of the Lie algebra. Furthermore combining the derivative of Eqn. (4) and the variation of $\widetilde{\mathbf{u}}$ yields a relationship that can be written in terms of vectors in $\mathbb{R}^{k}$

$$
\delta(\mathbf{u})=\frac{d(\delta \mathbf{q})}{d s}+\widehat{\mathbf{u}} \delta \mathbf{q}
$$

where $\widehat{\bullet}$ is a linear operator which maps a vector in $\mathbb{R}^{k}$ into a $k \times k$ matrix.

Eqn. (5) can be seen as a differential equation on the Lie group and its solution when $\widetilde{\mathbf{u}}$ does not depend on $s$ is

$$
q(s)=q_{0} \exp (s \tilde{\mathbf{u}})
$$

where $q_{0}=q(0)$ and exp is the exponential operator which maps an element of the Lie algebra to an element of the Lie group

$$
\exp : \mathfrak{g} \rightarrow G, \tilde{\mathbf{x}} \rightarrow \exp (\widetilde{\mathbf{x}})
$$


As the Lie algebra is isomorphic to $\mathbb{R}^{k}$, the exponential map introduces a local parametrization of the Lie group around any $q_{0} \in G$. Indeed, any $q \in G$ may be represented as a function of $\tilde{\mathbf{x}} \in \mathfrak{g}$ using the exponential operator and the composition with $q_{0}$ according to

$$
q=q_{0} \exp (\tilde{\mathbf{x}})
$$

The exponential map has the series development in terms of the Lie algebra element

$$
\exp (\widetilde{\mathbf{x}})=\sum_{i=0}^{\infty} \frac{\widetilde{\mathbf{x}}^{i}}{i !}
$$

By extension the notation $\exp (\mathbf{x})$ is equivalent since the Lie algebra is isomorphic to $\mathbb{R}^{k}$. The comparison of the derivative with respect to $s$ of Eqn. (9) with Eqn. (5) yields a linear relationship from $\mathbb{R}^{k}$ to $\mathbb{R}^{k}$

$$
\mathbf{u}=\mathbf{T}(\mathbf{x}) \frac{d \mathbf{x}}{d s}
$$

where $\mathbf{T}(\mathbf{x})$ is called the tangent application of the exponential. Likewise we can write

$$
\delta \mathbf{q}=\mathbf{T}(\mathbf{x}) \delta \mathbf{x}
$$

The tangent application has a series expansion given by

$$
\mathbf{T}(\mathbf{x})=\sum_{i=0}^{\infty}(-1)^{i} \frac{\widehat{\mathbf{x}}^{i}}{(i+1) !}
$$

The inverse of the exponential map is called the logarithmic map and is defined as

$$
\log (q): G \rightarrow \mathfrak{g}, \log (q) \rightarrow \widetilde{\mathbf{x}}
$$

The series development of the logarithmic map is

$$
\log (q)=\sum_{i=1}^{\infty}(-1)^{i+1} \frac{(q-e)^{i}}{i}
$$

\section{The Special Orthogonal group, $S O(3)$}

The special orthogonal group, $S O(3)$, is a matrix Lie group that can be represented by $3 \times 3$ orthogonal matrices $\mathbf{R}$ whose determinant is positive, that is $\operatorname{det}(\mathbf{R})=1$. In particular, rotation matrices belong to $S O(3)$. The neutral element is $\mathbf{I}_{3 \times 3}$ and the inverse of an element $\mathbf{R} \in S O(3)$ is $\mathbf{R}^{T} \in S O(3)$, where $\bullet^{T}$ is the matrix transpose. The composition rule is the matrix product. The Lie algebra, $\mathfrak{s o}(3)$, is the space of skew-symmetric matrices and is isomorphic to $\mathbb{R}^{3}$

$$
\boldsymbol{\Omega}=\left[\begin{array}{l}
\Omega_{1} \\
\Omega_{2} \\
\Omega_{3}
\end{array}\right] \in \mathbb{R}^{3} \text { and } \tilde{\boldsymbol{\Omega}}=\left[\begin{array}{ccc}
0 & -\Omega_{3} & \Omega_{2} \\
\Omega_{3} & 0 & -\Omega_{1} \\
-\Omega_{2} & \Omega_{1} & 0
\end{array}\right] \in \mathfrak{s o}(3)
$$


In particular Eqn. (4) reads

$$
\delta \mathbf{R}=\mathbf{R} \delta \tilde{\boldsymbol{\Theta}}
$$

The $\hat{\bullet}$ operator defined in Eqn. (6) is equivalent to $\widetilde{\bullet}$. The exponential map has a compact form given by Rodrigue's formula

$$
\exp _{S O(3)}(\boldsymbol{\Omega})=\mathbf{I}_{3 \times 3}+\frac{\sin (\|\boldsymbol{\Omega}\|)}{\|\boldsymbol{\Omega}\|} \tilde{\boldsymbol{\Omega}}+\frac{1-\cos (\|\boldsymbol{\Omega}\|)}{\|\boldsymbol{\Omega}\|^{2}} \widetilde{\boldsymbol{\Omega}}^{2}
$$

The tangent application is given by

$$
\mathbf{T}_{S O(3)}(\boldsymbol{\Omega})=\mathbf{I}_{3 \times 3}+\frac{\cos (\|\boldsymbol{\Omega}\|)-1}{\|\boldsymbol{\Omega}\|^{2}} \widetilde{\boldsymbol{\Omega}}+\frac{\|\boldsymbol{\Omega}\|-\sin (\|\boldsymbol{\Omega}\|)}{\|\boldsymbol{\Omega}\|^{3}} \widetilde{\boldsymbol{\Omega}}^{2}
$$

and the logarithmic map reads

$$
\log _{S O(3)}(\mathbf{R})=\tilde{\boldsymbol{\omega}}=\frac{\theta}{2 \sin (\theta)}\left(\mathbf{R}-\mathbf{R}^{T}\right)
$$

with $\theta=\operatorname{acos}\left(\frac{1}{2}(\operatorname{trace}(\mathbf{R})-1)\right),|\theta|<\pi$. If $\mathbf{R}=\mathbf{I}_{3 \times 3}, \theta=0$ and $\tilde{\boldsymbol{\omega}}=\mathbf{0}_{3 \times 3}$.

The Special Euclidean group, $S E(3)$

The matrix Lie group, $S E(3)$, is the group of homogeneous transformations $\mathbf{H}=$ $\mathcal{H}(\mathbf{R}, \mathbf{x})$, i.e. containing a rotation $\mathbf{R} \in S O(3)$ and a position vector $\mathbf{x} \in \mathbb{R}^{3}$. They can be represented by $4 \times 4$ matrices

$$
\mathbf{H}=\left[\begin{array}{cc}
\mathbf{R} & \mathbf{x} \\
\mathbf{0}_{1 \times 3} & 1
\end{array}\right]
$$

The neutral element is $\mathbf{I}_{4 \times 4}$ and the inverse of $\mathbf{H} \in S E(3)$ is $\mathbf{H}^{-1} \in S E(3)$ given by $\mathbf{H}^{-1}=\mathcal{H}\left(\mathbf{R}^{T},-\mathbf{R}^{T} \mathbf{x}\right)$. The composition rule is the matrix product. The Lie algebra, $\mathfrak{s e}(3)$, is the space of $4 \times 4$ matrices such as Eqn. (22) and is isomorphic to $\mathbb{R}^{6}$

$$
\mathbf{h}=\left[\begin{array}{l}
\mathbf{h}_{U} \\
\mathbf{h}_{\Omega}
\end{array}\right] \in \mathbb{R}^{6} \quad \text { and } \quad \tilde{\mathbf{h}}=\left[\begin{array}{cc}
\widetilde{\mathbf{h}_{\Omega}} & \mathbf{h}_{U} \\
\mathbf{0}_{1 \times 3} & 0
\end{array}\right] \in \mathfrak{s e}(3)
$$

where $\widetilde{\mathbf{h}_{\Omega}} \in \mathfrak{s o}(3)$ and $\mathbf{h}_{U} \in \mathbb{R}^{3}$. It is clear from the argument whether the tilde operator denotes the mapping to $\mathfrak{s o}(3)$ or $\mathfrak{s e}(3)$. The $\widehat{\bullet}$ operator defined in Eqn. (6) is

$$
\widehat{\mathbf{h}}=\left[\begin{array}{cc}
\widetilde{\mathbf{h}_{\Omega}} & \widetilde{\mathbf{h}}_{U} \\
\mathbf{0}_{3 \times 3} & \widetilde{\mathbf{h}_{\Omega}}
\end{array}\right]
$$

Eqn. (4) reads

$$
\delta \mathbf{H}=\mathbf{H} \delta \tilde{\mathbf{h}} \Leftrightarrow\left[\begin{array}{cc}
\delta \mathbf{R} & \delta \mathbf{x} \\
\mathbf{0}_{1 \times 3} & 1
\end{array}\right]=\left[\begin{array}{cc}
\mathbf{R} & \mathbf{x} \\
\mathbf{0}_{1 \times 3} & 1
\end{array}\right]\left[\begin{array}{cc}
\delta \widetilde{\mathbf{h}_{\Omega}} & \delta \mathbf{h}_{U} \\
\mathbf{0}_{1 \times 3} & 1
\end{array}\right]
$$


in which $\delta \mathbf{h}_{U}=\mathbf{R}^{T} \delta \mathbf{x}$ and $\delta \tilde{\mathbf{h}}_{\Omega}=\mathbf{R}^{T} \delta \mathbf{R}$. Accordingly, one has $\delta \mathbf{h}^{T}=\left[\begin{array}{ll}\delta \mathbf{h}_{U}^{T} & \delta \mathbf{h}_{\Omega}^{T}\end{array}\right]^{T}$. The exponential map is given by

$$
\exp _{S E(3)}(\mathbf{h})=\left[\begin{array}{cc}
\exp _{S O(3)}\left(\mathbf{h}_{\Omega}\right) & \mathbf{A}\left(\mathbf{h}_{\Omega}\right) \mathbf{h}_{U} \\
\mathbf{0}_{3 \times 1} & 1
\end{array}\right]
$$

where $\mathbf{A}\left(\mathbf{h}_{\Omega}\right)=\sum_{i=0}^{\infty}{\widetilde{\mathbf{h}_{\Omega}}}^{i} /(i+1) !=\mathbf{T}_{S O(3)}^{T}\left(\mathbf{h}_{\Omega}\right)$. The tangent application is given by

$$
\mathbf{T}_{S E(3)}(\mathbf{h})=\left[\begin{array}{cc}
\mathbf{T}_{S O(3)}\left(\mathbf{h}_{\Omega}\right) & \mathbf{A}_{1}\left(\mathbf{h}_{U}, \mathbf{h}_{\Omega}\right)-\frac{1}{2} \tilde{\mathbf{h}}_{U} \\
\mathbf{0}_{3 \times 3} & \mathbf{T}_{S O(3)}\left(\mathbf{h}_{\Omega}\right)
\end{array}\right]
$$

where $\mathbf{A}_{1}\left(\mathbf{h}_{U}, \mathbf{h}_{\Omega}\right)$ has the explicit form

$$
\begin{aligned}
& \frac{1-\beta}{2} \tilde{\mathbf{h}}_{U}+\frac{1-\alpha}{\left\|\mathbf{h}_{\Omega}\right\|^{2}}\left(\tilde{\mathbf{h}}_{U} \tilde{\mathbf{h}}_{\Omega}+\tilde{\mathbf{h}}_{\Omega} \tilde{\mathbf{h}}_{U}\right)-\frac{\alpha-\beta}{\left\|\mathbf{h}_{\Omega}\right\|^{2}}\left(\mathbf{h}_{\Omega}^{T} \mathbf{h}_{U}\right) \tilde{\mathbf{h}}_{\Omega} \\
& +\frac{1}{\left\|\mathbf{h}_{\Omega}\right\|^{2}}\left(\frac{\beta}{2}-\frac{3}{\left\|\mathbf{h}_{\Omega}\right\|^{2}}(1-\alpha)\right)\left(\mathbf{h}_{\Omega}^{T} \mathbf{h}_{U}\right) \tilde{\mathbf{h}}_{\Omega} \tilde{\mathbf{h}}_{\Omega}
\end{aligned}
$$

with $\alpha=\sin \left(|| \mathbf{h}_{\Omega}||\right) / \| \mathbf{h}_{\Omega}||$ and $\beta=2\left(1-\cos \left(|| \mathbf{h}_{\Omega}||\right)\right) /\left\|\mathbf{h}_{\Omega}\right\|^{2}$. Notice that $\mathbf{A}_{1}\left(\mathbf{h}_{U}, \mathbf{h}_{\Omega}\right)$ vanishes if $\mathbf{h}_{\Omega}=\mathbf{0}$. The logarithmic map reads

$$
\log _{S E(3)}(\mathbf{H})=\left[\begin{array}{cc}
\tilde{\boldsymbol{\omega}} & \mathbf{A}^{-1}(\boldsymbol{\omega}) \mathbf{x} \\
\mathbf{0}_{1 \times 3} & 0
\end{array}\right]
$$

where $\tilde{\boldsymbol{\omega}}=\log _{S O(3)}(\mathbf{R})$.

\section{BEAM FINITE ELEMENT FORMULATION}

We address the formulation of a straight beam element on $S E(3)$ using a finite element method based on an exponential interpolation. We develop first the kinematics, then the deformation measure and finally the internal forces from the virtual work principle. The developments are particularized to the small deformation and linear elasticity context.

\section{Beam kinematics}

Defining $s$ as the parameter along the neutral axis of the element, $\mathbf{x}^{0}(s)$ is the position vector of a point of the neutral axis in the reference configuration (see Fig. 1). Thus, the position of any point $p$ of the beam in the reference configuration can be written as

$$
\mathbf{x}_{p}^{0}(s, t, u)=\mathbf{x}^{0}(s)+t \mathbf{i}_{t}+u \mathbf{i}_{u}
$$

where $\mathbf{i}_{t}$ and $\mathbf{i}_{u}$ are the principal axes of the cross-sections, and $t$ and $u$ are the coordinates

of these axes. We assume that the principal axes are constant along the neutral axis, which 


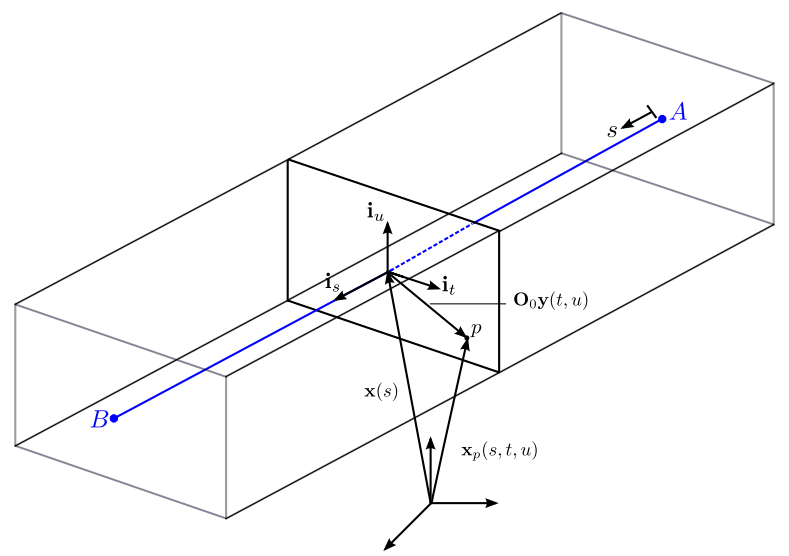

FiguRE 1 - Beam in the reference configuration, with point $A$ at $s=0$ and point $B$ at $s=L$.

means that the beam is initially straight.

We introduce the following notation

$$
\mathbf{x}_{p}^{0}(s, t, u)=\mathbf{x}^{0}(s)+\mathbf{O}_{0} \mathbf{y}(t, u)
$$

in which $\mathbf{y}(t, u)=\left[\begin{array}{lll}0 & t & u\end{array}\right]^{T}, \mathbf{O}_{0}=\left[\begin{array}{lll}\mathbf{i}_{s} & \mathbf{i}_{t} & \mathbf{i}_{u}\end{array}\right]$ and $\mathbf{i}_{s}$ is the unit vector along the neutral axis. $\mathbf{O}_{0}$ is a rotation matrix that accounts for the orientation of the reference configuration of the beam with respect to the inertial frame. We assume that the cross-sections remain undeformed, which means $\mathbf{y}(t, u)$ does not depend on the configuration. By extension, the position of a point $p$ in the current configuration can be written as

$$
\mathbf{x}_{p}(s, t, u)=\mathbf{x}(s)+\mathbf{R}(s) \mathbf{O}_{0} \mathbf{y}(t, u)
$$

where $\mathbf{x}(s)$ is the position vector of the neutral axis and $\mathbf{R}(s)$ characterizes the rotation of the cross-sections.

In the $S E(3)$ framework the neutral axis on the beam is described by a mapping $\mathbb{R} \rightarrow$ $S E(3): s \rightarrow \mathbf{H}(s)$. Accordingly, the neutral axis of the beam element is described by $\mathbf{H}(s)=\mathcal{H}\left(\mathbf{I}_{3 \times 3}, \mathbf{x}^{0}(s)\right)$ in the reference configuration and $\mathbf{H}(s)=\mathcal{H}(\mathbf{R}(s), \mathbf{x}(s))$ in the current configuration. Based on Eqn. (31) the configuration of the beam at any point $p$ of coordinates $(s, t, u)$ is given by

$$
\mathbf{H}_{p}(s, t, u)=\mathbf{H}(s)\left[\begin{array}{cc}
\mathbf{I}_{3 \times 3} & \mathbf{O}_{0} \mathbf{y}(t, u) \\
\mathbf{0}_{1 \times 3} & 1
\end{array}\right]=\mathbf{H}(s) \mathbf{H}_{\mathbf{y}}(t, u)
$$

where $\mathbf{H}_{p}(s, t, u)=\mathcal{H}\left(\mathbf{R}_{p}(s), \mathbf{x}_{p}(s, t, u)\right)$ and $\mathbf{R}_{p}(s)=\mathbf{R}(s)$ since the beam kinematics assumes that the cross-sections do not deform, namely they remain straight. Hence, $\mathbf{H}_{\mathbf{y}}(t, u)$ does not depend on the configuration but $\mathbf{H}(s)$ does. 


\section{Beam finite element discretization}

Similarly to linear space formulation, the discretization of the neutral axis of the beam is introduced by an interpolation between two end nodes $A$ at $s=0$ and $B$ at $s=L$ with a variable $s \in[0, L]$ along the neutral axis and $L$ is the length of the element. Using the $S E(3)$ framework, we introduce an interpolation based on the exponential formula

$$
\mathbf{H}(s)=\mathbf{H}_{A} \exp _{S E(3)}\left(\frac{s}{L} \widetilde{\mathbf{h}}\right)
$$

where we define the relative configuration vector $\tilde{\mathbf{h}}=\log _{S E(3)}\left(\mathbf{H}_{A}^{-1} \mathbf{H}_{B}\right)$ that is invariant under rigid body motions.

Notice that in the reference configuration $\mathbf{H}_{A}=\mathcal{H}\left(\mathbf{I}_{3 \times 3}, \mathbf{x}_{A}^{0}\right)$ and $\mathbf{H}_{B}=\mathcal{H}\left(\mathbf{I}_{3 \times 3}, \mathbf{x}_{B}^{0}\right)$ such that $\mathbf{h}_{U}=\mathbf{x}_{B}^{0}-\mathbf{x}_{A}^{0}$ and $\mathbf{h}_{\Omega}=\mathbf{0}_{3 \times 1}$. Hence, the rotation field over the element in the reference configuration is $\mathbf{R}(s)=\mathbf{I}_{3 \times 3}$. The configuration of the beam is readily obtained introducing Eqn. (33) into Eqn. (32).

\section{Beam deformation measure}

The deformations of the neutral axis are introduced from the deformation gradient of the material frame on the neutral axis. Owing to the Lie group derivative, the deformation gradient is a Lie algebra element denoted as $\widetilde{\mathbf{f}} \in \mathfrak{s e}(3)$ and is defined from

$$
\frac{d \mathbf{H}(s)}{d s}=\mathbf{H}(s) \tilde{\mathbf{f}}
$$

f can be split into two contributions

$$
\mathbf{f}=\mathbf{f}^{0}+\boldsymbol{\epsilon}
$$

where $\mathbf{f}^{0}$ refers to the values in the reference (undeformed) configuration and $\boldsymbol{\epsilon}$ accounts for the deformations. Thus, $\boldsymbol{\epsilon}$ vanishes in the reference configuration. $\boldsymbol{\epsilon}$ has the structure $\left[\begin{array}{ll}\gamma^{T} & \boldsymbol{\kappa}^{T}\end{array}\right]^{T}$ where $\boldsymbol{\gamma}$ and $\boldsymbol{\kappa} \in \mathbb{R}^{3}$. Considering Eqn. (33), the derivative of the interpolation formula reads

$$
\frac{d \mathbf{H}(s)}{d s}=\mathbf{H}(s)\left(\mathbf{T}\left(\frac{s}{L} \tilde{\mathbf{h}}\right) \frac{\mathbf{h}}{L}\right)=\mathbf{H}(s) \frac{\tilde{\mathbf{h}}}{L}
$$

and, comparing to Eqn. (34), we have $\mathbf{f}=\mathbf{h} / L, \mathbf{f}_{U}=\mathbf{h}_{U} / L$ and $\mathbf{f}_{\Omega}=\mathbf{h}_{\Omega} / L$. Consequently, the deformations are computed as

$$
\begin{aligned}
\gamma & =\frac{\mathbf{h}_{U}-\mathbf{h}_{U}^{0}}{L} \\
\kappa & =\frac{\mathbf{h}_{\Omega}}{L}
\end{aligned}
$$


The deformations are thus constant over the length of the element. The deformations at any point of the cross-section can be computed from the derivative with respect to $s$ of (32), which leads to

$$
\frac{\partial \mathbf{H}_{p}(s, t, u)}{\partial s}=\mathbf{H}_{p}(s, t, u) \tilde{\mathbf{f}}_{p}
$$

in which $\tilde{\mathbf{f}}_{p}=\mathbf{H}_{\mathbf{y}}^{-1} \tilde{\mathbf{f}} \mathbf{H}_{\mathbf{y}}$, such that

$$
\mathbf{f}_{p}=\left[\begin{array}{c}
\mathbf{f}_{p U} \\
\mathbf{f}_{\Omega}
\end{array}\right]
$$

where $\mathbf{f}_{p U}=\mathbf{f}_{U}-\widetilde{\mathbf{O}_{0} \mathbf{y}} \mathbf{f}_{\Omega}=\left(\mathbf{h}_{U}-\widetilde{\mathbf{O}_{0} \mathbf{y}} \mathbf{h}_{\Omega}\right) / L$. According to Eq. (35), we can write $\mathbf{f}_{p}=\mathbf{f}_{p}^{0}+\boldsymbol{\epsilon}_{p}$ where $\boldsymbol{\epsilon}_{p}=\left[\begin{array}{ll}\gamma_{p} & \boldsymbol{\kappa}\end{array}\right]$ with $\gamma_{p}=\mathbf{f}_{p U}-\mathbf{f}_{p U}^{0}=\gamma-\widetilde{\mathbf{O}_{0} \mathbf{y} \boldsymbol{\kappa}}=\left(\mathbf{h}_{U}-\mathbf{h}_{U}^{0}-\widetilde{\mathbf{O}_{0} \mathbf{y} \mathbf{h}_{\Omega}}\right) / L$. Let us now consider the metric tensors in order to build deformation tensors. $\mathbf{g}$, the metric tensor in the current configuration, is defined as

$$
\mathbf{g}_{i j}=\left(\frac{\partial \mathbf{x}_{p}(s, t, u)}{\partial i}\right)^{T} \frac{\partial \mathbf{x}_{p}(s, t, u)}{\partial j}
$$

with $i, j=\{s, t, u\}$. Using Eqn. (31) and Eqn. (40) to develop the derivatives, we have

$$
\begin{aligned}
\frac{\partial \mathbf{x}_{p}(s, t, u)}{\partial s} & =\mathbf{R}(s) \mathbf{f}_{p U} \\
\frac{\partial \mathbf{x}_{p}(s, t, u)}{\partial t} & =\mathbf{R}(s) \mathbf{i}_{t} \\
\frac{\partial \mathbf{x}_{p}(s, t, u)}{\partial u} & =\mathbf{R}(s) \mathbf{i}_{u}
\end{aligned}
$$

The polar decomposition of the deformation gradient $\mathbf{F}=\mathbf{R U}=\mathbf{R}\left[\begin{array}{lll}\mathbf{f}_{p U} & \mathbf{i}_{t} & \mathbf{i}_{u}\end{array}\right]$ is straightforward. The metric tensor in the reference configuration, denoted $\mathbf{g}^{0}$, has the same definition as $\mathbf{g}$, but involves $\mathbf{x}_{p}^{0}, \mathbf{f}_{p U}^{0}$ and $\mathbf{R}(s)=\mathbf{I}_{3 \times 3}$. Notice that due to the assumption that the cross-sections remain underformed, $\mathbf{i}_{t}$ and $\mathbf{i}_{u}$ are identical in any configuration.

The Green-Lagrange strain tensor $\mathbf{G L}_{i j}=\frac{1}{2}\left(\mathbf{g}_{i j}-\mathbf{g}_{i j}^{0}\right)$ can now be computed. It involves three kinds of terms

$$
\begin{aligned}
\mathbf{G L}_{s s} & =\frac{1}{2}\left(\mathbf{f}_{p U}^{T} \mathbf{f}_{p U}-\mathbf{f}_{p U}^{0 T} \mathbf{f}_{p U}^{0}\right) \\
\mathbf{G L}_{s i} & =\frac{1}{2} \boldsymbol{\gamma}_{p}^{T} \mathbf{i}_{i} \\
\mathbf{G L}_{i j} & =0
\end{aligned}
$$

with $i, j=\{t, u\}$. $\mathbf{G L}_{i j}$ vanish and $\mathbf{G L}_{s i}$ takes into account the cross-section are undeformable. Also, assuming that the deformations $\boldsymbol{\gamma}$ and $\boldsymbol{\kappa}$ are small, we can linearize the second order term $\mathbf{f}_{p U}^{T} \mathbf{f}_{p U}$ in $\mathbf{G L}_{s s}$ (Eqn. (45)) as $2 \gamma_{p}^{T} \mathbf{f}_{p U}^{0}+\mathbf{f}_{p U}^{0 T} \mathbf{f}_{p U}^{0}$ and we obtain

$$
\mathbf{G L}_{s s}=\gamma_{p}^{T} \mathbf{f}_{p U}^{0}
$$


It is remarkable that the Green-Lagrange strain tensor has a simple compact form and is constant over the length of the element.

\section{Internal Forces}

The virtual work principle

$$
\delta(\mathcal{W})=\int_{V} \mathbf{S}: \delta(\mathbf{G L}) d V
$$

where $\mathbf{S}$ is the second Piola-Kirchhoff stress tensor, allows to computed the internal forces of the finite element. The variation of the Green-Lagrange strain tensor, defined in Eqn. (4548 ), involves only the variation of $\boldsymbol{\gamma}$ and $\boldsymbol{\kappa}$ (through $\boldsymbol{\gamma}_{p}$ ). Owing to the fact that $\boldsymbol{\gamma}$ and $\boldsymbol{\kappa}$ are constant over the cross-section, we obtain

$$
\delta \mathcal{W}=\int_{L}\left(\delta(\gamma)^{T} \mathbf{n}+\delta(\boldsymbol{\kappa})^{T} \mathbf{m}\right) d s
$$

where $\mathbf{n}$ and $\mathbf{m}$ are respectively the resulting forces and momenta over the cross-sections. For a linear elastic material the constitutive law can be expressed as

$$
\left[\begin{array}{c}
\mathbf{n} \\
\mathbf{m}
\end{array}\right]=\underbrace{\left[\begin{array}{cc}
\mathbf{C}_{U} & \mathbf{0}_{3 \times 3} \\
\mathbf{0}_{3 \times 3} & \mathbf{C}_{\Omega}
\end{array}\right]}_{=\mathbf{C}}\left[\begin{array}{l}
\boldsymbol{\gamma} \\
\kappa
\end{array}\right]
$$

where $\mathbf{C}$ contains the usual stiffness parameters. It depends on the constitutive law and is obtained from an integration over the cross-section. For simple cases, it is diagonal and $\mathbf{C}_{U}=\mathbf{O}_{0} \operatorname{diag}\left(E A, G A_{t}, G A_{u}\right) \mathbf{O}_{0}^{T}$ contains the axial and shear stiffnesses while $\mathbf{C}_{\Omega}=$ $\mathbf{O}_{0} \operatorname{diag}\left(G J, E I_{t}, E I_{u}\right) \mathbf{O}_{0}^{T}$ contains the torsional and bending stiffnesses. Notice that this analysis of the constitutive law and of the shape of the cross-sections must be carried out only once (in the reference configuration) and the resulting constitutive relationship is valid in any configuration.

Using the definition (33) and (36), the variation of $\mathbf{h}$ and of $\mathbf{f}$ can be expressed in terms of variation $\delta \mathbf{h}_{A B}=\left[\begin{array}{ll}\delta \mathbf{h}_{A}^{T} & \delta \mathbf{h}_{B}^{T}\end{array}\right]^{T}$, the $12 \times 1$ virtual increment vector with $\delta \mathbf{h}_{A}=$ $\left[\begin{array}{ll}\delta \mathbf{h}_{U A}^{T} & \delta \mathbf{h}_{A \Omega}^{T}\end{array}\right]^{T}, \delta \mathbf{h}_{B}=\left[\begin{array}{ll}\delta \mathbf{h}_{U B}^{T} & \delta \mathbf{h}_{B \Omega}^{T}\end{array}\right]^{T}$ as

$$
\delta(\boldsymbol{\epsilon})=\delta\left(\frac{\mathbf{h}}{L}\right)=\frac{\mathbf{P}(\mathbf{h})}{L} \delta \mathbf{h}_{A B}
$$

in which $\mathbf{P}(\mathbf{h})=\left[-\mathbf{T}_{S E(3)}(-\mathbf{h})^{-1} \quad \mathbf{T}_{S E(3)}(\mathbf{h})^{-1}\right]$ is constant over the element. We can now substitute the variation of the deformations with the virtual displacements (Eqn. (52)) in Eqn. (50) to obtain the internal forces $\mathbf{f}_{\text {int }}$

$$
\delta \mathcal{W}=\delta \mathbf{h}_{A B}^{T} \mathbf{P}(\mathbf{h})^{T} \mathbf{C}\left[\begin{array}{l}
\gamma \\
\kappa
\end{array}\right]=\delta \mathbf{h}_{A B}^{T} \mathbf{f}_{i n t}(\mathbf{h})
$$


The integration over the length can be performed exactly since all the terms are constant over the length. The tangent stiffness matrix is given by

$$
\mathbf{K}_{T}=\underbrace{\frac{1}{L} \mathbf{P}(\mathbf{h})^{T} \mathbf{C P}(\mathbf{h})}_{=\mathbf{K}_{m}}+\underbrace{\frac{d \mathbf{P}(\mathbf{h})^{T}}{d \mathbf{h}_{A B}} \mathbf{C}\left[\begin{array}{l}
\boldsymbol{\Gamma} \\
\boldsymbol{\kappa}
\end{array}\right]}_{=\mathbf{K}_{g}}
$$

The so-called material part $\mathbf{K}_{m}$ is obtained straightforwardly and the so-called geometric part $\mathbf{K}_{g}$ can be neglected without impeding the numerical convergence in case of small deformations.

We observe that the internal force and the tangent stiffness matrix can be obtained by exact integration over the element and that they are expressed in compact form. Moreover $\mathbf{P}, \boldsymbol{\gamma}$ and $\boldsymbol{\kappa}$ depend on $\mathbf{H}_{A}$ and $\mathbf{H}_{B}$ only through the relative configuration vector $\mathbf{h}$. Consequently, $\mathbf{f}_{\text {int }}$ and $\mathbf{K}_{T}$ only depend on $\mathbf{h}$ and are thus invariant under rigid body motions.

\section{A FEW GOOD REASONS TO CONSIDER THE PROPOSED FORMULATION}

Several observations are now discussed in order to highlight some important features of the proposed formulation.

\section{Natural coupling and non-linear interpolations}

Classical beam theories imply that position and the rotation variables are kinematically coupled and the equations of mechanics developed under such kinematic assumptions yield in general coupled differential equations that govern both the position and the rotation variables. Consequently, the cross-sections rotate under a shear load and a bending moment involves a displacement of the neutral axis. However, the position and the rotation variables are treated as independent variables as well as their increments in usual finite element discretization schemes. It is not the case with the proposed formulation. Indeed, the position of a point of the neutral axis computed via the interpolation formula (33) reads

$$
\mathbf{x}(s)=\mathbf{x}_{A}+\mathbf{R}_{A} \mathbf{A}\left(\frac{s}{L} \mathbf{h}_{\Omega}\right) \frac{s}{L} \mathbf{h}_{U}
$$

where we observe that both the parts $\mathbf{h}_{U}$ and $\mathbf{h}_{\Omega}$ of the relative configuration vectors are involved. It means that the coupling is deeper compared to usual formulations. Moreover, the interpolation of the positions in Eqn. (55) is strongly non-linear due to the $\mathbf{A}$ matrix from the exponential map. The interpolation of the rotations is also non-linear and reads

$$
\mathbf{R}(s)=\mathbf{R}_{A} \exp \left(\frac{s}{L} \tilde{\mathbf{h}}_{\Omega}\right)
$$


which is identical to the formula used by Crisfield and Jelenic [12].

According to Eqn. (55), the interpolation method allows to describe a non-linear displacement field. In order to discuss the geometry of the displacement field, let us computed the curvature $k$ of the neutral axis using the classical formula

$$
k=\left\|\frac{d \mathbf{t}(s)}{d s}\right\|=\frac{\left\|\tilde{\mathbf{h}}_{\Omega} \mathbf{h}_{U}\right\|}{L}
$$

since

$$
\frac{d}{d s}(\mathbf{t}(s))=\frac{d}{d s}\left(\frac{d \mathbf{x}}{d s}\left\|\frac{d \mathbf{x}}{d s}\right\|^{-1}\right)=\mathbf{R}(s) \frac{\tilde{\mathbf{h}}_{\Omega} \mathbf{h}_{U}}{L}
$$

where $\mathbf{t}(s)$ is the unit vector which is tangent to the curve. We observe that the curvature is a constant over the element. Therefore the proposed interpolation method allows to represent exactly an element of constant curvature thanks to the non-linear coupling between the rotation part and the position part in Eqn. (55).

\section{Absence of shear locking}

The finite element formulation of shear deformable beam, usually called Timoshenko or Cosserat beam theory, with linear interpolation and exact integration exhibit a shear locking phenomenon [1]. It can be discussed by considering the shear in a cantilever beam submitted to a bending moment at its free end. Theoretically, the shear strain should vanish since pure bending does not produce any shear force. Numerically, such an element subjected to pure bending undergoes spurious shear deformations, the shear energy does not vanish and a too stiff behaviour is observed. The problem can classically be removed by a reduced integration method that filters high order bending contribution to shear. The proposed shear deformable beam finite element turns out to be shear locking-free. This can be observed by inspecting the tangent stiffness matrix of one element in a straight configuration, that is $\mathbf{h}_{\Omega}=\mathbf{0}$,

$$
\mathbf{K}_{T}=\frac{1}{L}\left[\begin{array}{cccc}
\mathbf{C}_{U} & -\frac{\mathbf{C}_{U} \widetilde{\mathbf{h}}_{U}}{2} & -\mathbf{C}_{U} & -\frac{\mathbf{C}_{U} \widetilde{\mathbf{h}}_{U}}{2} \\
\frac{\widetilde{\mathbf{h}_{U}} \mathbf{C}_{U}}{2} & \mathbf{C}_{\Omega}-\frac{\widetilde{\mathbf{h}}_{U} \mathbf{C}_{U} \widetilde{\mathbf{h}}_{U}}{2} & -\frac{\widetilde{\mathbf{h}}_{U} \mathbf{C}_{U}}{2} & -\mathbf{C}_{\Omega}-\frac{\widetilde{\mathbf{h}}_{U} \mathbf{C}_{U} \widetilde{\mathbf{h}}_{U}}{4} \\
-\mathbf{C}_{U} & \frac{\mathbf{C}_{U} \widetilde{\mathbf{h}}_{U}}{2} & \mathbf{C}_{U} & \frac{\mathbf{C}_{U} \widetilde{\mathbf{h}}_{U}}{2} \\
\frac{\widetilde{\mathbf{h}}_{U} \mathbf{C}_{U}}{2} & -\mathbf{C}_{\Omega}-\frac{\widetilde{\mathbf{h}}_{U} \mathbf{C}_{U} \widetilde{\mathbf{h}}_{U}}{4} & -\frac{\widetilde{\mathbf{h}}_{U} \mathbf{C}_{U}}{2} & \mathbf{C}_{\Omega}-\frac{\widetilde{\mathbf{h}}_{U} \mathbf{C}_{U} \widetilde{\mathbf{h}}_{U}}{4}
\end{array}\right]
$$

It appears that this matrix is similar to the one obtained by reduced integration of standard shear deformable beam formulation. In particular, there is no energy contribution of the shear stiffness when computing the energy in pure bending, that is $0.5 \mathbf{B}^{T} \mathbf{K}_{T} \mathbf{B}$, where $\mathbf{B}=\left[\begin{array}{lll}\mathbf{0}_{3 \times 1}^{T} & \mathbf{b}^{T} & \mathbf{0}_{3 \times 1}^{T}-\mathbf{b}^{T}\end{array}\right], \mathbf{b}=\left[\begin{array}{lll}0 & b_{t} & b_{u}\end{array}\right]^{T}$. Hence the proposed formulation exhibits naturally a shear locking-free behaviour for bending about a straight configuration. Notice that the residual bending flexibility correction can be applied to our formulation [1] such 
that the exact solution of linear theory in displacement is recovered. When the beam element is deformed, the terms in $\mathbf{K}_{T}$ change, and a more involved discussion is necessary to prove the absence of locking. This will be done in a further work.

We compared the proposed formulation to the one presented in [1] for the large displacement case of a cantilever beam (see Fig. 2). A $5 \mathrm{~m}$ long beam is subjected to a vertical force of $6 e 5 \mathrm{~N}$ at its free end. The properties of the beam are the cross-section area $A=4.8 e-3$ $\mathrm{m}^{2}$, the bending stiffness $E I=9.345 e 6 \mathrm{Nm}^{2}$ and the shear stiffness $k G A=3.213 e 8 \mathrm{Nm}^{2}$. The residual bending flexibility correction was taken into account. The reference solution was computed with 600 elements. Both methods yield a very close result for such number of elements. The comparison in Fig. 3 indicates that the proposed formulation leads to better accuracy for small number of elements.

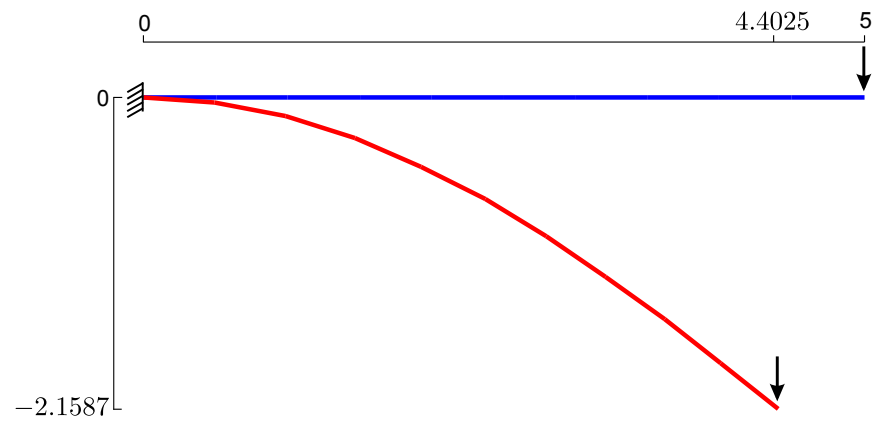

Figure 2 - Cantilever beam subjected to a vertical load at is free end.

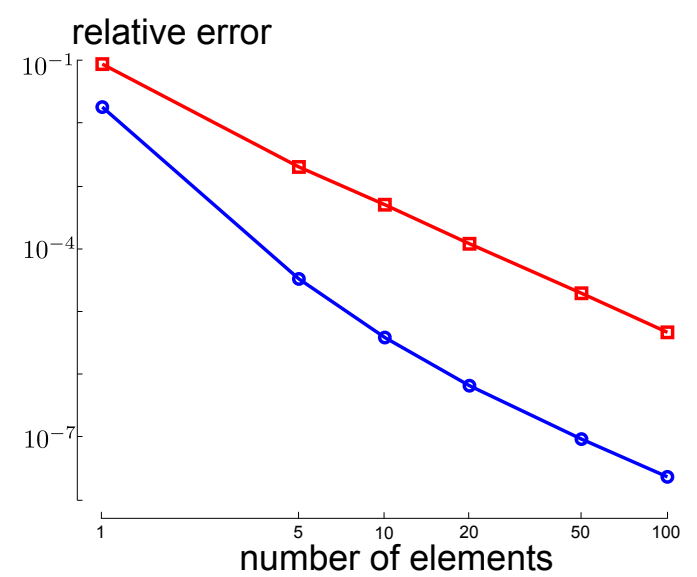

FiguRE 3 - Relative error on the vertical displacement of the free end obtained with the proposed formulation (blue circles) and with the reference (red squares). 


\section{Exact displacement field in pure bending}

Let us consider a planar cantilever beam submitted to a bending moment at its free end. From strength of material, the solution is known to be a pure bending deformation and a neutral axis of constant curvature. Here, we show that the proposed method provides the geometrically exact result in pure bending. Let us consider that the beam is initially aligned with the $x$-axis of the inertial frame in the reference configuration and a moment $M$ is applied about the $z$-axis (see Fig. 4). The exact solution in pure bending reads

$$
\begin{aligned}
x(s) & =\frac{1}{k} \sin (s k) \\
y(s) & =\frac{1}{k}(1-\cos (s k)) \\
z(s) & =0
\end{aligned}
$$

where $k=M /(E I)$ is the curvature. Regarding our formulation, the reference configuration is characterized by $\mathbf{h}_{U}=\left[\begin{array}{lll}L & 0 & 0\end{array}\right]^{T}$ and $\mathbf{h}_{\Omega}=\left[\begin{array}{lll}0 & 0 & 0\end{array}\right]^{T}$. In pure bending, the neutral axis does not undergo any axial or shear deformations such that $\mathbf{h}_{U}$ does not change, however the neutral axis gets a curvature $k$ in the $x y$-plan and we have $\mathbf{h}_{\Omega}=L\left[\begin{array}{lll}0 & 0 & k\end{array}\right]^{T}$. Introducing these in the non-linear interpolation field in Eqn. (55) turns out to yield the exact solution given in Eqn. (60-62). The sin and cos functions come from the A operator of the exponential.

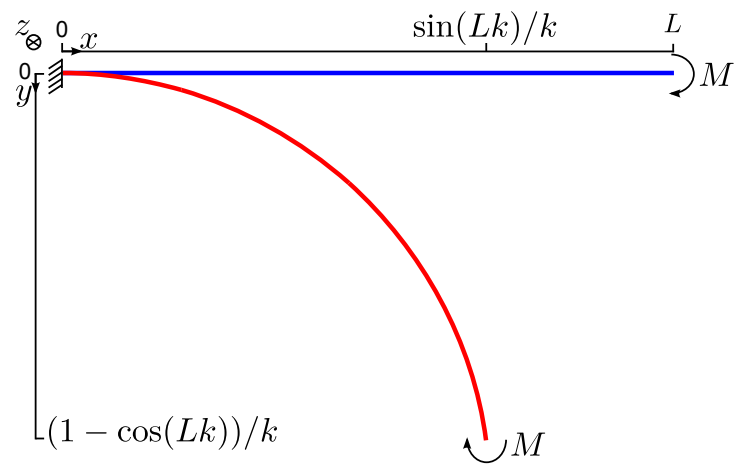

Figure 4 - Cantilever beam subjected to a moment $M$ at its free end. The reference configuration is initially horizontal and the deformed configuration is a planar curve of constant curvature.

In previous sections we discussed that the proposed formulation is shear locking-free and that the non-linear formula for the interpolation of position was such that the element is able the represent exactly a curve of constant curvature. Since the exact solution of the planar cantilever beam problem is a curve of constant curvature the finite element solution matches it exactly even with one single element. 


\section{Invariant tangent stiffness matrix under rigid body motions}

As mentioned earlier, the relative configuration vector $\mathbf{h}$ in Eqn. (33) is invariant under rigid body motions and so is the tangent stiffness matrix $\mathbf{K}_{T}$ in Eqn. (54). In order to reduce computational costs in small deformation context, we suggest to keep the tangent stiffness matrix computed in the reference configuration for a whole simulation. The number of iterations might increase since we neglect the deformation contributions, but the iteration matrix can be assembled and inverted only once for the whole simulation. In order to illustrate the advantage, let us consider a stiff horizontal beam connected to a wall by a small rotational stiffness and subjected to a small vertical load at its free end. The position at the wall is fixed. As a numerical example, the beam is made of steel, $1 \mathrm{~m}$ long and has a square cross-section of area $0.01 \mathrm{~m}^{2}$, the rotation stiffness is $100 \mathrm{~N} /$ radians and the vertical load is $50 \mathrm{~N}$. The beam is discretized with 5 elements. The solution is plotted in Fig. 5. The beam undergoes large displacements but small deformations and the solution was obtained using a constant tangent stiffness matrix namely the tangent stiffness matrix computed in the reference configuration.

If the deformations are not negligible a non-systematic update procedure of the tangent stiffness matrix could be considered to reduce computational costs.

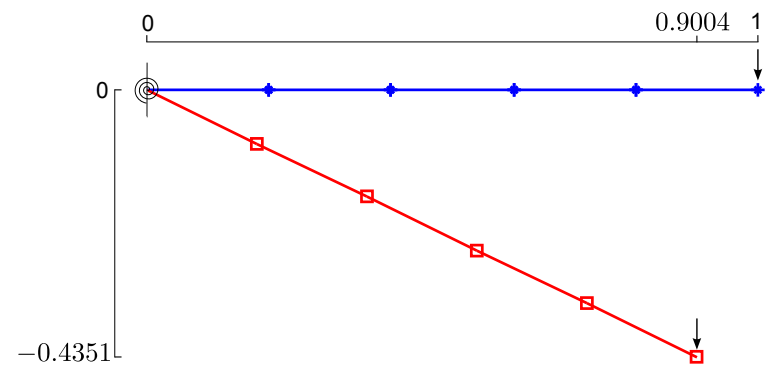

FIGURE 5 - Large displacement and small deformation of a stiff beam connected to a wall by a small rotational stiffness and subjected to a small vertical load at its free end.

\section{CONCLUSIONS AND PERSPECTIVES}

We have presented a straight beam finite element formulation on $S E(3)$ based on a non-linear interpolation method. The $S E(3)$ framework allows well defined and compact notations, and introduces a natural coupling between the position and the rotation variables.

The proposed beam finite element formulation exhibits some important features. The deformation terms are clearly separated from the global motion terms such that rigid body motions do not come into play in the evaluation of the elastic forces and the tangent stiffness matrix. The interpolation formula leads to constant deformation measures over the length 
of the element. Consequently, the internal forces and the tangent stiffness matrix can be integrated exactly over the length of the element and are expressed in compact forms.

Some particular situations were discussed to present the advantages of the proposed method. It was shown that the proposed method is naturally shear locking-free. We also showed that the exact displacement field in case of pure bending can be obtained with one single element thanks to the non-linear interpolation of the position field due to the $S E(3)$ framework.

The authors think that numerical evaluation procedures of the tangent stiffness matrix could be investigated in order to fully benefit from the invariance under rigid body motions. Dynamic formulation of the beam element could also benefit much from the proposed framework. It would also be interesting to extend the proposed method to curved beam elements or plate and shell elements. Higher order polynomials in the argument of the exponential map might also be investigated.

The first author would like to acknowledge the Belgian National Fund for Scientific Research for its financial support (FRIA fellowship).

\section{Références}

[1] M. Géradin and A. Cardona. Flexible Multibody Dynamics : A Finite Element Approach. John Wiley \& Sons, Chichester, 2001.

[2] J.C. Simo. A finite strain beam formulation. The three-dimensional dynamic problem. Part I. Computer Methods in Applied Mechanics and Engineering, 49 :55-70, 1985.

[3] A. A. Shabana and R. Y. Yakoub. Three dimensional absolute nodal coordinate formulation for beam elements : Theory. Journal of Mechanical Design, 123(4) :606-613, 2001.

[4] I. Romero. A comparison of finite elements for nonlinear beams : the absolute nodal coordinate and geometrically exact formulations. Multibody System Dynamics, 20(1) :51-68, 2008.

[5] P. Betsch and P. Steinmann. Frame-indifferent beam finite elements based upon the geometrically exact beam theory. International Journal for Numerical Methods in Engineering, 54 :1775-1788, 2002.

[6] P. Jung, S. Leyendecker, J. Linn, and M. Ortiz. A discrete mechanics approach to the cosserat rod theory - part 1 : static equilibria. International Journal for Numerical Methods in Engineering, 85 :31-60, 2011.

[7] O. Brüls, A. Cardona, and M. Arnold. Lie group generalized- $\alpha$ time integration of constrained flexible multibody systems. Mechanism and Machine Theory, 48 :121$137,2012$. 
[8] O. Brüls, M. Arnold, and A. Cardona. Two Lie group formulations for dynamic multibody systems with large rotations. In Proceedings of the IDETC/MSNDC Conference, Washington D.C., U.S., August 2011.

[9] O. Sander. Geodesic finite elements for Cosserat rods. International Journal for $\mathrm{Nu}$ merical Methods in Engineering, 82 :1645-1670, 2010.

[10] R. M. Murray, Z. Li, and S. S. Sastry. A mathematical introduction to robotic manipulation. CRC Press, March 1994.

[11] A. Cardona and M. Géradin. A beam finite element non-linear theory with finite rotations. International Journal for Numerical Methods in Engineering, 26 :2403-2438, 1988.

[12] M.A. Crisfield and G. Jelenic. Objectivity of strain measures in the geometrically exact three-dimensional beam theory and its finite-element implementation. Proceedings of the Royal Society of London A, 455 :1125-1147, 1999.

[13] F.C. Park. Distance metrics on the rigid-body motions with applications to mechanism design. Transactions of the ASME, 117 :48-54, 1995.

[14] M. Zefran and V. Kumar. Interpolation schemes for rigid body motions. ComputerAided Design, 30(3) :179-189, 1998.

[15] W.M. Boothby. An Introduction to Differentiable Manifolds and Riemannian Geometry. Academic Press, 2nd edition, 2003. 